\title{
Revisiting the $\mathrm{OH}+\mathrm{H}_{2} \rightarrow \mathrm{H}_{2} \mathrm{O}+\mathrm{H}$ reaction at the molecular level: the plausible catalytic role of ice in its own reconstruction
}

\author{
P. Redondo ${ }^{1,2}$, F. Pauzat ${ }^{1}$, A. Markovits ${ }^{1}$, and Y. Ellinger ${ }^{1}$ \\ ${ }^{1}$ Sorbonne Universités, UPMC Univ. Paris 06, UMR, CNRS 7616, Laboratoire de Chimie Théorique, 75252 Paris Cedex 05, France \\ e-mail: yves.ellinger@sorbonne-universite.fr \\ ${ }^{2}$ Universidad de Valladolid, Facultad de Ciencias (Dpto. QF), Valladolid, Spain \\ e-mail: predondo@qf.uva.es
}

Received 4 August 2020 / Accepted 26 December 2020

\begin{abstract}
Context. In spite of the permanent damage suffered from the radiation field (cosmic rays, X-rays, and intense UV-visible radiations), interstellar grains are still covered by ices mantles whose role in interstellar chemistry is well beyond any doubt. This clearly means that the destruction of the ice cover has to be counterbalanced by efficient reconstruction mechanisms.

Aims. Our goal is to determine whether the ice, which is still present after irradiation, has a catalytic role in the $\mathrm{OH}+\mathrm{H}_{2} \rightarrow \mathrm{H}_{2} \mathrm{O}+\mathrm{H}$ reaction for its own reconstruction. We focus on the three plausible reaction paths depending on the way reactants $\mathrm{OH}_{\text {or }} \mathrm{H}_{2}$ are adsorbed at the ice surface.

Methods. Calculations were performed in both cluster and solid state approaches, using ab-initio post Hartree-Fock methods for small systems, standard density functional theory (DFT) for larger clusters, and periodic solid state DFT with specific formalisms accounting for weak interactions in systems of infinite dimensions.

Results. Although the end product is the same, that is namely the reconstruction of one $\mathrm{H}_{2} \mathrm{O}$ on the subjacent ice, three different reaction paths are found depending on whether $\mathrm{H}_{2}$ reacts with adsorbed $\mathrm{OH}(\mathrm{ads})$, wether $\mathrm{OH}$ reacts with adsorbed $\mathrm{H}_{2}$ (ads) or wether both $\mathrm{OH}(\mathrm{ads})$ and $\mathrm{H}_{2}$ (ads) are adsorbed on the ice before reacting. In the first case, there is an activation barrier of $\sim 6 \mathrm{kcal} \mathrm{mol}^{-1}$, requiring the tunneling effect for the reaction to proceed, which is in agreement with preceding studies. In the second case, the reaction is a barrierless process leading to the direct reconstruction of the ice. In the third case, the double adsorption increases the activation barrier due to the lowering of the starting energy. This is found regardless of the dimension of the supporting ice aggregates.

Conclusions. Icy grain surfaces play a critical role for their own reconstruction in cold, dense interstellar clouds. The prevalence of tunneling over the direct mechanism should strongly depend on the temperature and local environment.
\end{abstract}

Key words. astrochemistry - ISM: molecules - ISM: abundances - molecular processes - solid state: volatile

\section{Introduction}

The hypothesis of the existence of water ice in the interstellar medium (ISM) goes back to the beginning of the last century when Eddington (1926) proposed that the water molecules would aggregate to form small grains in the dark clouds between stars. Then, infrared observations have shown that ice is a robust material that can be found almost everywhere in the cold ISM (Merrill et al. 1976; Whittet, et al. 1988; Dartois et al. 1998; Gibb et al. 2004; Boogert et al. 2008; Oberg et al. 2011). Moreover, a general consensus has developed that processes on icy grains had to play a major role in astrochemistry. A thorough presentation of the history of water formation can be found in Oba et al. (2012). The key reference is that of Tielens \& Hagen (1982) followed by numerous studies (d'Hendecourt et al. 1985; Hasegawa \& Herbst 1993; Cuppen \& Herbst 2007), a non-exhaustive list that can be extended to the present days with Meisner et al. (2017) and Lamberts, \& Kästner (2017).

Considering the extremely dilute environment of the ISM, it is agreed that a simple freeze out of the water present in the gas phase cannot be the only origin of the icy grain mantles in which $\mathrm{H}_{2} \mathrm{O}$ is the dominant component. The problem becomes even more sensitive if one considers that the ice mantles are currently subject to intense damages from the radiation field (irradiation by cosmic rays, energetic $\mathrm{X}$-rays, $\mathrm{UV}$-visible radiations, etc.), resulting in continuous destruction of the ices, which have to be reconstructed one way or another by surface reactions. Two irrefutable pieces of evidence have to be taken into account. On the one hand, the rupture of the $\mathrm{H}_{2} \mathrm{O}$ molecules gives three elementary fragments, that is $\mathrm{H}, \mathrm{O}$, and $\mathrm{OH}$, all of these free radicals are known to be particularly reactive. On the other hand, the grain surface is widely covered by $\mathrm{H}_{2}$ which is the dominant species in the environment.

Looking at the available reactants, the most plausible reactions, that is to say those that effectively yield $\mathrm{H}_{2} \mathrm{O}$, are as follows:

$\mathrm{H}+\mathrm{OH} \rightarrow \mathrm{H}_{2} \mathrm{O}$

$\mathrm{H}_{2}+\mathrm{OH} \rightarrow \mathrm{H}_{2} \mathrm{O}+\mathrm{H}$

to which, according to Cuppen \& Herbst (2007), a third reaction should be added,

$\mathrm{H}_{2} \mathrm{O}_{2}+\mathrm{H} \rightarrow \mathrm{H}_{2} \mathrm{O}+\mathrm{OH}$.

From their kinetic model Cuppen \& Herbst evaluated the efficiency of the above three reactions to be 6,77 and $17 \%$ at $10 \mathrm{k}$ in dense clouds for (1), (2) and (3), respectively. Formation of $\mathrm{H}_{2} \mathrm{O}$ following

$\mathrm{OH}+\mathrm{OH} \rightarrow \mathrm{H}_{2} \mathrm{O}+\mathrm{O}$ 
has also been recently considered as plausible when supported by ice layers (Redondo et al. 2020). This reaction appeared to be a barrierless mechanism that could be more important than thought originally. It has to be noted that the formation of $\mathrm{OH}$ could formally appear in

$\mathrm{H}+\mathrm{O} \rightarrow \mathrm{OH}$

but this process should be most likely marginal, due to the volatility of the hydrogen atom. Diffusion of $\mathrm{H}$ atoms might also be an other reason of the presence of the molecular $\mathrm{H}_{2}$ that is used by reaction (2). More is presented in the chemical network proposed by Tielens \& Hagen (1982) for $\mathrm{H}_{2} \mathrm{O}$ synthesis. For example, hydrogenation of atomic oxygen, molecular oxygen (Ioppolo et al. 2010; Cuppen et al. 2010) and ozone (Mokrane et al. 2009) are not only at the origin of several chemical models but also inspired most of the laboratory astrophysics studies.

The important facts are that the $\mathrm{OH}$ radical appears at one stage or another in all mechanisms of $\mathrm{H}_{2} \mathrm{O}$ formation and that $\mathrm{H}_{2}$ is omnipresent in the icy grains environment. These are two main reasons why we focused on reaction (2). Even if the end products are the same, i.e. formation of a water molecule on the icy surface and release of a $\mathrm{H}$ atom, three reaction mechanisms are to be considered at the molecular level. The differences rely on the distinct structures of their respective adsorption elementary steps

$\mathrm{H}_{2}+\mathrm{OH}_{\mathrm{ads}} \rightarrow \mathrm{H}_{2} \mathrm{O}_{\mathrm{ads}}+\mathrm{H}$
$\mathrm{OH}+\mathrm{H}_{2}$ ads $\rightarrow \mathrm{H}_{2} \mathrm{O}_{\mathrm{ads}}+\mathrm{H}$
$\left[\mathrm{OH}+\mathrm{H}_{2}\right]_{\mathrm{ads}} \rightarrow \mathrm{H}_{2} \mathrm{O}_{\mathrm{ads}}+\mathrm{H}$.

On the one hand, the well-admitted reaction of $\mathrm{H}_{2}$ with an adsorbed $\mathrm{OH}_{\text {ads }}$ radical, on another hand the reaction of $\mathrm{OH}$ with an adsorbed $\mathrm{H}_{2}$ ads and finally the reaction starting with the reagents $\left[\mathrm{OH}+\mathrm{H}_{2}\right]_{\text {ads }}$ both adsorbed on the surface or in a void inside the ice.

\section{Computational methods}

Looking for the feasibility of the $\mathrm{OH}+\mathrm{H}_{2}$ reaction on a solid water ice support requires procedures capable of giving comparable descriptions of the reactive potential energy profile whatever the extension of the substrate support (aggregate of finite or solid of infinite dimensions). If ab-initio methods can be used for clusters with small numbers of $\mathrm{H}_{2} \mathrm{O}$ molecules, DFT methods are the natural choice for large structures and solids.

\subsection{Cluster approach}

In cluster calculations, the correlation and dispersion effects were taken into account in post Hartree-Fock MP2 and coupled cluster calculations using single, double and triple excitations within the CCSD and CCSD(T) formalism (Bartlett \& Shavitt 1977; Raghachavari et al. 1989). Then, anticipating calculations on much larger systems, we considered also the BHandHLYP (Becke 1993) and the MPW1K and MPWB1K functionals (Lynch 2000; Zao \& Truhlar 2004). Concerning basis sets, we have essentially employed the cc-pVTZ basis sets, augmented with diffuse functions. For the largest calculations we used the 6-311++G(3df,2p).

Each structure, fully optimized, was verified to be a stationary point by vibrational analysis. We checked also that including entropic terms to determine Gibbs free energies of formation at the temperature of the interstellar grains considered here has
Table 1. Relative energies ( $\left.\mathrm{kcal} \mathrm{mol}^{-1}\right)$ of critical points along the reaction profile for $\mathrm{H}_{2}\left({ }^{1} \Sigma^{+}\right)+\mathrm{OH}\left({ }^{2} \Pi\right) \rightarrow \mathrm{H}_{2} \mathrm{O}\left({ }^{1} \mathrm{~A}_{1}\right)+\mathrm{H}\left({ }^{2} \mathrm{~S}\right)$, including zero point vibrational energies.

\begin{tabular}{lccc}
\hline \hline Level of theory ${ }^{(*)}$ & $\mathrm{H}_{2}+\mathrm{OH}$ & $\mathrm{TS}$ & $\mathrm{H}_{2} \mathrm{O}+\mathrm{H}$ \\
\hline CCSD(T)/aug-T & 0.0 & 6.06 & -13.22 \\
MP2/aug-T & 0.0 & 7.91 & -21.94 \\
CCSD(T)/aug-T//MP2/aug-T & 0.0 & 6.12 & -13.47 \\
MPWB1K/aug-T & 0.0 & 4.77 & -13.39 \\
MPW1K/aug-T & 0.0 & 5.46 & -12.04 \\
BHandHLYP/aug-T & 0.0 & 9.33 & -7.00 \\
\hline
\end{tabular}

Notes. ${ }^{(*)}$ Basis set: /aug-T $=$ aug-cc-pVTZ.

a marginal impact on the values of the energy differences. All the calculations were performed using methods and basis sets as implemented in the GAUSSIAN09 package (Frisch et al. 2013).

\subsection{Solid state approach}

As in previous works on interactions of organic molecules with water ices (Lattelais et al. 2011, 2015; Redondo et al. 2020) we used the plane waves Vienna ab initio simulation package (VASP; Kresse \& Hafner 1993, 1994) to carry out all the solid state calculations. The treatment makes use of the same (PBE+D2) functionals corrected following Grimme et al. (2010) to account for the long range interactions specific of that type of studies and to which $50 \%$ of exact Hartree-Fock exchange was added to make comparison possible with MPW1K and MPWB1K functionals. Details on the ice modeling are presented in Appendix A.

\section{The $\mathrm{H}_{2}+\mathrm{OH}$ gas phase reaction as a case study}

Using the gas phase reaction as a test, we compare the CCSD(T) values with those of DFT calculations with the same basis sets. Energies for critical points are presented in Table 1.

At the $\operatorname{CCSD(T)/aug-T~level,~which~we~consider~as~level~}$ of reference, it is an exothermic process with a value of $\Delta H$ $\sim 13-14 \mathrm{kcal} \mathrm{mol}^{-1}$. It is opposed by an energy barrier $\Delta H^{\#}$ $\sim 6 \mathrm{kcal} \mathrm{mol}^{-1}$ in agreement with the literature (Chen et al. 2013). As in our previous study of the $\mathrm{OH}+\mathrm{OH}$ reaction (Redondo et al. 2020) we found that the stationary points are the same for all levels of theory and that the whole reaction proceeds in the same plane for all methods.

Compared to $\operatorname{CCSD}(\mathrm{T}) /$ aug-T, the exothermicity and activation barrier are largely overestimated at the MP2 level. It shows that higher excitations are clearly needed to get reasonable values as already found for $\mathrm{OH}+\mathrm{OH}$. However, using the MP2 optimized geometries in the CCSD(T)/aug-T//MP2/aug-T calculations is a less expansive alternative since it provides energy differences similar to those obtained in fully optimized CCSD(T)/aug-T treatments. Concerning DFT methods, the newly developed MPW1K/MPWB1K functionals provide energies lower in the average by $\sim 0.7 \mathrm{kcal} \mathrm{mol}^{-1}$ for exothermicity and activation barriers than optimized $\operatorname{CCSD}(\mathrm{T})$, these values being most reasonable for methods a priori not calibrated for small radicals reactions.

The first conclusion to be drawn from this case study approach is that a reasonable geometry can be obtained at the MP2 level that can be employed for the calculation of the energy profile at higher level of theory $(\operatorname{CCSD}(\mathrm{T}))$, 


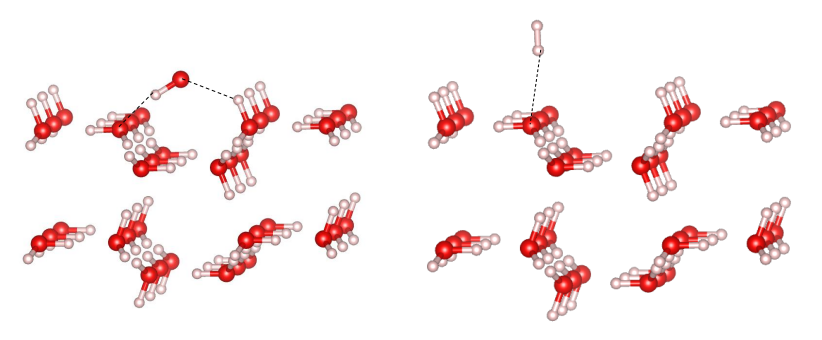

Fig. 1. Adsorption of $\mathrm{OH}$ (left) and $\mathrm{H}_{2}(r i g h t)$ on ice; the same arrangements are found (not shown) for large clusters 24(1-L.) and 48(2-L.).

The second conclusion concerns the larger calculations for which the DFT approach can be limited to MPW1K $\left(\Delta H^{\#}\right) / \mathrm{MPWB} 1 \mathrm{~K}(\Delta H)$ functionals in cluster type calculations. This test model, that is only relevant to gas phase, is clearly impeded by an activation barrier of $\sim 6 \mathrm{kcal} \mathrm{mol}^{-1}$. A similar result has been found by Meisner et al. (2017) using $\mathrm{QM} / \mathrm{MM}$ semi-empirical simulations, that is a reaction barrier of $5.74 \mathrm{kcal} \mathrm{mol}^{-1}$.

At this point, it should be emphasized that $\operatorname{CCSD}(\mathrm{T})$ is only considered to validate the use of MPW1K/MPWB1K functionals to follow the adsorption process from the simplest aggregates to larger molecular clusters formed by increasing the number $n$ of $\mathrm{H}_{2} \mathrm{O}$ molecules up to the $48 \mathrm{H}_{2} \mathrm{O}$ in structures composed of two bi-layers. These calculations employ the current type of Gaussian atom-localized basis sets. They are not intended to select the functional to be used for a solid state of infinite dimensions. In this latter case, the determination of the binding energies makes use of DFT $(\mathrm{PBE}+\mathrm{D} 2)$ periodic calculations using plane-wave basis sets, thus insuring by construction the continuity of the electronic density in the entire solid, a continuity that cannot be obtained, even for $\operatorname{CCSD}(\mathrm{T})$, with atomic basis sets of tractable dimensions. In fact, we have used two different approaches adapted to two different molecular scales. More details on the aptitude of the periodic "first principle" approach to the calculation of gas-molecular solid interactions can be found in Doronin et al. (2015) and Bertin et al. (2017).

\section{Interstellar context: $\mathrm{OH}$ versus $\mathrm{H}_{2}$ adsorption}

The ice that is destroyed is essentially that which covers the interstellar grains. As long as the temperature is not high enough to vaporize the mantle completely, there is a possibility for the ice to play the role of a full partner in reaction (2). But one has to realize that the possibility for the ice to intervene as catalytic support in such a reaction raises a question that does not exist in gas, namely, which of the partners is present on the ice $(\mathrm{OH}$ or $\mathrm{H}_{2}$ or both). How differently the reaction might proceed is critically dependent of their respective binding energies to the ice support and abundances.

The most stable geometries are presented in Fig. 1 for the case of solid water ice. The binding energies along the series of the aggregates considered for the simulations are given in Table 2.

The $\mathrm{OH}$ radical is positioned as a bridge on the surface (Fig. 1-left), with two hydrogen bonds. One of these bonds implies the hydrogen atom and an electronic lone pair of a surface oxygen, the other a lone pair of the oxygen and an hydrogen of a dangling $\mathrm{OH}$ bond of the surface. This double link leads to an interaction energy $\mathrm{E}(\mathrm{OH})_{\mathrm{ads}}=16.1 \mathrm{kcal} \mathrm{mol}^{-1}$ with the solid
Table 2. Electronic binding energies ${ }^{(*)}\left(\mathrm{kcal} \mathrm{mol}^{-1}\right)$ of $\mathrm{H}_{2}$ ads and $\mathrm{OH}_{\mathrm{ads}}$ as a function of the number $(n)$ of $\mathrm{H}_{2} \mathrm{O}$ in the supporting cluster.

\begin{tabular}{lcccccc}
\hline \hline Cluster $(n)$ & 1 & 2 & 3 & $24(1-\mathrm{L})$. & $48(2-\mathrm{L})$. & $\infty$ (Solid) \\
\hline $\mathrm{E}(\mathrm{OH})_{\mathrm{ads}}$ & 5.6 & 9.7 & 10.3 & 12.5 & 12.0 & 16.1 \\
$\mathrm{E}\left(\mathrm{H}_{2}\right)_{\mathrm{ads}}$ & 0.5 & 0.5 & 0.5 & 1.4 & 1.1 & 1.7 \\
\hline
\end{tabular}

Notes. ${ }^{(*)}$ MPWB1K/aug-cc-pVTZ except for the 2-layers case where the basis set is 6-311++G(3df,2p). The PBE+D2 functional, coupled with plane waves basis set has been used for solid state descriptions.

surface much stronger than that with a single $\mathrm{H}_{2} \mathrm{O}$ or even with the larger clusters $\left(5.6\right.$ to $\sim 12 \mathrm{kcal} \mathrm{mol}^{-1}$ ) as shown in Table 2 .

The $\mathrm{H}_{2}$ molecule has only one hydrogen bond (Fig. 1-right), this being collinear with an electronic lone pair of a surface oxygen. The minimum distance between two adjacent $\mathrm{H}_{2}$ ads molecules on the surface is $\sim 4.4 \AA$, which is larger than the equilibrium distance in the $\mathrm{H}_{2}$ dimer. Thus, contrary to the adsorption of $\mathrm{H}_{2}$ on graphene (Pauzat et al. 2011) there is no lateral interactions (attractive) to stabilize the coverage of ice by molecular hydrogen ${ }^{1}$.

From these results (Table 2) it can be stated that:

(i) When increasing the dimensions of the supporting cluster layer models, both $\mathrm{E}\left(\mathrm{H}_{2}\right)_{\text {ads }}$ and $\mathrm{E}(\mathrm{OH})_{\text {ads }}$ tend toward limits consistent with those obtained in the solid representation.

(ii) The binding energies $\mathrm{E}\left(\mathrm{H}_{2}\right)_{\mathrm{ads}}$ on the small clusters $(n=1-3)$ are rather small for the eventual variations be hidden within the computational accuracy; only the larger clusters reveal the global binding energy increase as in the $\mathrm{OH}$ case.

(iii) For the binding energy, $\mathrm{E}(\mathrm{OH})_{\mathrm{ads}}$, the first leap from 5.6 to $9.7 \mathrm{kcal} \mathrm{mol}^{-1}$ when going from a one to a two-molecule support shows the change from one to two hydrogen bonds; the following increases, smaller, illustrate a cooperative effect which culminates with the solid representation.

(iv) The $\mathrm{E}(\mathrm{OH})_{\text {ads }}$ binding energy is about one order of magnitude larger than $\mathrm{E}\left(\mathrm{H}_{2}\right)_{\text {ads }}$ whatever the dimension of the solid support.

(v) On the other side, $\mathrm{E}\left(\mathrm{H}_{2}\right)_{\text {ads }}$ can effectively stay attached on the water-ice surface only in cold environments in which the mantle of the grains is surrounded mostly by molecular hydrogen.

(vi) Considering the binding energies in Table 2 it is more than plausible that both $\mathrm{OH}_{\text {ads }}$ and $\mathrm{H}_{2 \text { ads }}$ can coexist on the ice surface, which in the end opens the way to a third mechanism. In the end we face three possibilities for the reconstruction of the ice, namely, reaction of $\mathrm{H}_{2}$ on $\mathrm{OH}_{\text {ads }}$, actually considered the dominant process, attack of the $\mathrm{OH}$ radical on $\mathrm{H}_{2}$ ads and reaction between $\mathrm{OH}_{\text {ads }}$ and $\mathrm{H}_{2}$ ads, both partners being already adsorbed or remaining trapped inside the irradiated ice. The first two belong to the Eley-Rideal type, (hereafter referred to as $\mathrm{ER}\left(\mathrm{OH}_{\mathrm{ads}}\right)$ and $\mathrm{ER}\left(\mathrm{H}_{2}\right.$ ads, $)$ respectively), whereas the third is a typical Langmuir- Hinschelwood, $\mathrm{LH}\left(\mathrm{OH}+\mathrm{H}_{2}\right)_{\text {ads }}$ mechanism.

\footnotetext{
1 With binding energies of $\sim 0.7-1.7 \mathrm{kcal} \mathrm{mol}^{-1}$ on ice (Govers et al. 1980; Perets et al. 2005; Burke \& Brown 2010; Amiaud et al. 2015) and $\sim 0.9 \mathrm{kcal} \mathrm{mol}^{-1}$ for graphene (average (Vidali et al. 1991; Pauzat et al. 2011) the surface number densities of $\mathrm{H}_{2}$ ads are close, namely, $0.3 \times 10^{15} \mathrm{~cm}^{-2}$ and $0.8 \times 10^{15} \mathrm{~cm}^{-2}$ on water ice and graphene, respectively. This ratio of $\sim 2.5$ is not correlated to the difference between the binding energies on these different surfaces but simply to the greater number of adsorption sites i.e. 6 nearest neighbors for graphene versus 2 for ice (Pauzat et al. 2011).
} 
Table 3. Relative energies $\left(\mathrm{kcal} \mathrm{mol}^{-1}\right)$ for the reaction profile for $\mathrm{H}_{2}\left({ }^{1} \Sigma^{+}\right)+\mathrm{OH}_{\mathrm{ads}}\left({ }^{2} \Pi\right) \rightarrow \mathrm{H}_{2} \mathrm{O}\left({ }^{1} \mathrm{~A}_{1}\right)+\mathrm{H}\left({ }^{2} \mathrm{~S}\right)$ as a function of the number of $\mathrm{H}_{2} \mathrm{O}$ molecules in the supporting cluster.

\begin{tabular}{|c|c|c|c|c|c|c|c|c|c|c|c|c|c|c|}
\hline \multirow{2}{*}{$\begin{array}{l}\text { Cluster size }(n) \\
\text { Level of theory }{ }^{(*)}\end{array}$} & \multicolumn{2}{|c|}{0} & \multicolumn{2}{|c|}{1} & \multicolumn{2}{|c|}{2} & \multicolumn{2}{|c|}{3} & \multicolumn{2}{|c|}{ 24(1-layer) } & \multicolumn{2}{|c|}{ 48(2-layers) } & \multicolumn{2}{|c|}{$\infty$ (Solid) } \\
\hline & $\Delta H^{\#}$ & $\Delta H$ & $\Delta H^{\#}$ & $\Delta H$ & $\Delta H^{\#}$ & $\Delta H$ & $\Delta H^{\#}$ & $\Delta H$ & $\Delta H^{\#}$ & $\Delta H$ & $\Delta H^{\#}$ & $\Delta H$ & $\Delta H^{\#}$ & $\Delta H$ \\
\hline CCSD(T)//MP2/-T & 5.7 & -15.0 & 5.8 & -14.4 & 5.5 & -15.8 & 5.2 & -16.3 & & & & & & \\
\hline MPWB1K/aug-T & 4.2 & -15.3 & 4.7 & -14.4 & 4.6 & -15.7 & 4.5 & -16.1 & 4.1 & -17.5 & 3.5 & -16.9 & & \\
\hline MPW1K/aug-T & 4.8 & -14.0 & 5.3 & -13.0 & 5.3 & -14.3 & 5.2 & -14.8 & 5.1 & -14.0 & 4.8 & -15.3 & & \\
\hline VASP/Plane wave & & & & & & & & & & & & & 4.1 & -16.2 \\
\hline
\end{tabular}

Notes. $\Delta \mathrm{H}^{\#}=$ transition state $\left[\left(\mathrm{H}_{2} \mathrm{O}\right)_{n} \ldots \mathrm{OH} \ldots . \mathrm{H}_{2}\right]$ energy and $\Delta \mathrm{H}=$ exothermicity of the reaction. ${ }^{(*)}$ Basis set for cluster supports: $/ \mathrm{T}=$ aug-ccpVTZ (except for the 2-layers structure where the basis set is the $6-311++\mathrm{G}(3 \mathrm{df}, 2 \mathrm{p})$ ); the reference energy is the electronic energy of OH adsorbed on the ice (except gas phase $(\boldsymbol{n}=0)$. The adsorption sites are locally optimized for the mono-layer and the bi-layer $\left(6\right.$ interacting $\left.\mathrm{H}_{2} \mathrm{O}\right)$.

\section{Adding $\mathrm{H}_{2}$ in the $\mathrm{OH}$ adsorbed model}

Knowing the difference in binding energies (one order of magnitude) between $\mathrm{H}_{2}$ and $\mathrm{OH}$ on an icy surface, we started with the case when the hydroxyl radical $\mathrm{OH}_{\mathrm{ads}}$ is already attached on the ice. Looking for consistency between the cluster and the solid state representations, we considered the possible role of neighboring molecules. To this end, we progressively increased the number $n$ of water molecules in the reaction support. Reporting the gas phase results $(n=0)$ for comparison, we present in Table 3 the evolution of the transition state energies $\Delta H^{\#}$ from $n=1-3$ to bi-layers of $\mathrm{H}_{2} \mathrm{O}$ molecules and in fine to a slab of ice of infinite dimension.

\subsection{The $\mathrm{H}_{2}+\mathrm{OH}_{\text {ads }}$ reaction atop small clusters}

In the presence of $\mathrm{OH}_{\text {ads }}$ adsorbed on one, two, then three $\mathrm{H}_{2} \mathrm{O}$ molecules, we obtain energy profiles very similar to that of the gas phase reaction. It is illustrated in Fig. 2 on the example of the reaction occurring on a water dimer support taking the MPWB1K energy reported in Table 3 . The reaction with $\mathrm{H}_{2}$ proceeds through transition states $\sim 5 \mathrm{kcal} \mathrm{mol}^{-1}$ above the starting complexes. The final products in these model reactions consist in a $\left[\left(\mathrm{H}_{2} \mathrm{O}\right)_{n+1}\right]$ structure showing the growth of the water support together with an hydrogen atom desorbing to the gas phase. Whatever the size of the small cluster the ice reconstruction are exothermic by $\sim 15-16 \mathrm{kcal} \mathrm{mol}^{-1}$ (Table 3 ). Up to this point, there is no clear-cut correlation to the number of $\mathrm{H}_{2} \mathrm{O}$ taken to model the ice support.

\subsection{The $\mathrm{H}_{2}+\mathrm{OH}_{\text {ads }}$ reaction atop ice layers}

It is well known that increasing the dimension of the cluster (that is to say increasing $n$ ) leads, after full geometry optimizations, to very different stable structures that do not properly mimic the solid water ice, as demonstrated by the numerical simulations (Buch et al. 2004) of the structures of $\left(\mathrm{H}_{2} \mathrm{O}\right)_{n}$ aggregates. Consequently, in order to go one step further in the modeling of the ice environment, we set up an $\mathrm{H}_{2} \mathrm{O}$ layer model. As in the previous study of $\mathrm{OH}+\mathrm{OH}$ reaction (Redondo et al. 2020) we take the topmost bi-layer of an apolar hexagonal ice $I_{\mathrm{h}}$ that has been shown to be the most stable surface (Pan et al. 2010) and only the reaction site was optimized in order to avoid the aforementioned artifacts.

For these larger models we used the MPWB1K/MPW1K functionals. However, the dimension of the system $\mathrm{H}_{2}+\mathrm{OH}$ $+\left[\mathrm{H}_{2} \mathrm{O}\right]$ bi-layers makes the geometry optimizations with the aug-cc-pVTZ basis set barely tractable. Therefore, we have

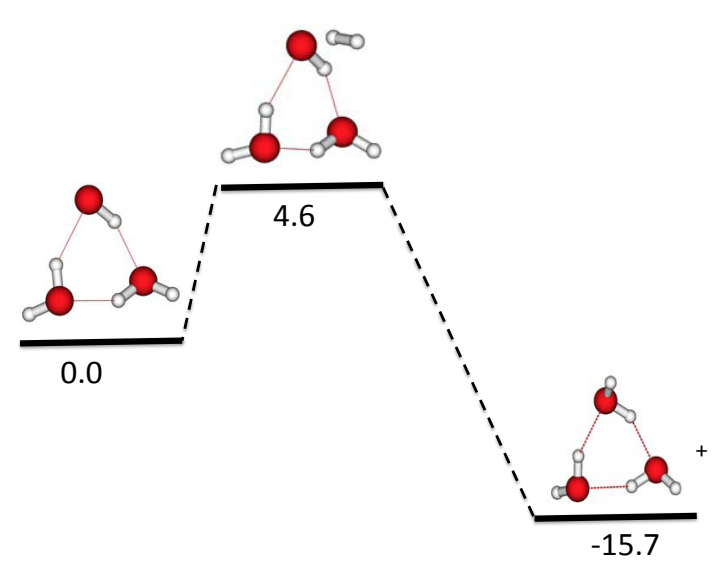

Fig. 2. Reaction profile for $\mathrm{H}_{2}\left({ }^{1} \Sigma^{+}\right)+\mathrm{OH}\left({ }^{2} \Pi\right) \rightarrow \mathrm{H}_{2} \mathrm{O}\left({ }^{1} \mathrm{~A}_{1}\right)+\mathrm{H}\left({ }^{2} \mathrm{~S}\right)$ at the MPWB1K/aug-cc-pVTZ level in presence of $\mathrm{H}_{2} \mathrm{O}$ dimer. Relative energies are given in $\mathrm{kcal} \mathrm{mol}^{-1}$.

characterized the critical points on the surface employing a smaller double zeta basis set augmented, as previously, by diffuse functions $\left(3-21+\mathrm{G}^{* *}\right)$. The energies were then refined by single points calculations using the larger aug-cc-pVTZ and 6$311++\mathrm{G}(3 \mathrm{df}, 2 \mathrm{p})$ basis sets ${ }^{2}$. The reaction profile of $\mathrm{H}_{2}+\mathrm{OH}$ supported by the water ice layer is presented in Fig. 3. Starting from $\mathrm{OH}_{\text {ads }}$ adsorbed on the ice surface, the reaction proceeds towards an $\mathrm{H}_{2} \mathrm{O}$ molecule attached to the surface with an hydrogen desorbing. Snapshots of the reaction site along the reaction path are presented for more details. The important point is that the energy barrier is still present on the reaction path and remains close to $\sim 5 \mathrm{kcal} \mathrm{mol}^{-1}$ ) even in a 2 bi-layer cluster model (Table 3).

\subsection{The $\mathrm{H}_{2}+\mathrm{OH}_{\text {ads }}$ reaction atop solid water ice}

The next step is to represent the environment of the reaction path by a solid icy surface of infinite dimension. The same reaction scheme as above is investigated, starting with the hydroxyl radical $\mathrm{OH}_{\mathrm{ads}}$ first adsorbed on the surface. Several stable positions of attachment are possible (Meisner et al. 2017). We opted for the energetically most stable, i.e. bridge conformation.

\footnotetext{
2 The $\operatorname{CCSD}(\mathrm{T})$ reaction path of $\mathrm{H}_{2}+\mathrm{OH}$ with three $\mathrm{H}_{2} \mathrm{O}$ molecules was correctly reproduced by this procedure. As an example, the transition state is located 5.10, 4.92 and $4.85 \mathrm{kcal} \mathrm{mol}^{-1}$ above the reference at the MPWB1K/aug-cc-pVTZ, MPWB1K/6$311++\mathrm{G}(3 \mathrm{df}, 2 \mathrm{p}) / / \mathrm{MPWB} 1 \mathrm{~K} / 3-21+\mathrm{G}^{* *}$ and MPWB1K/aug-cc-pVTZ// MPWB1K/3-21+G** levels, respectively.
} 


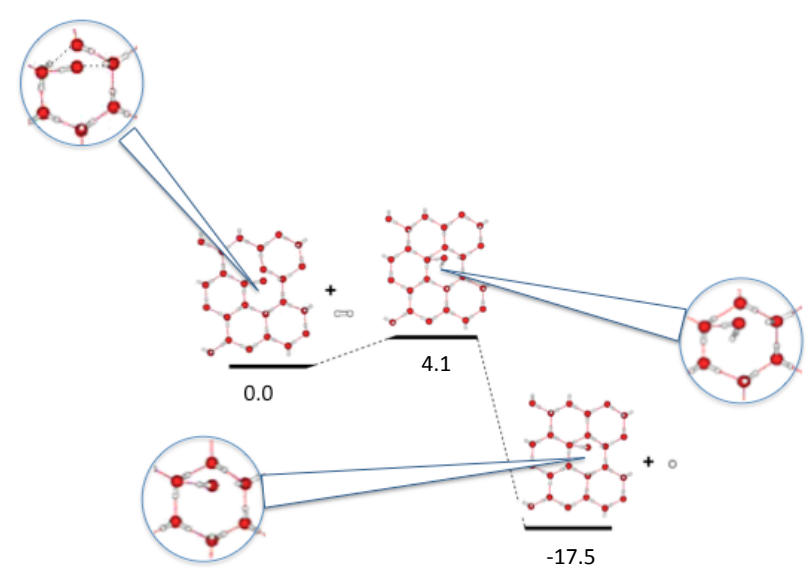

Fig. 3. Reaction profile for $\mathrm{H}_{2}\left({ }^{1} \Sigma^{+}\right)+\mathrm{OH}_{\mathrm{ads}}\left({ }^{2} \Pi\right) \rightarrow \mathrm{H}_{2} \mathrm{O}_{4 \mathrm{ads}}\left({ }^{1} \mathrm{~A}_{1}\right)+$ $\mathrm{H}\left({ }^{2} \mathrm{~S}\right)$ at the MPWB1K/aug-cc-pVTZ level in presence of a 1-layer of $\mathrm{H}_{2} \mathrm{O}$. Relative energies are given in $\mathrm{kcal} \mathrm{mol}^{-1}$.

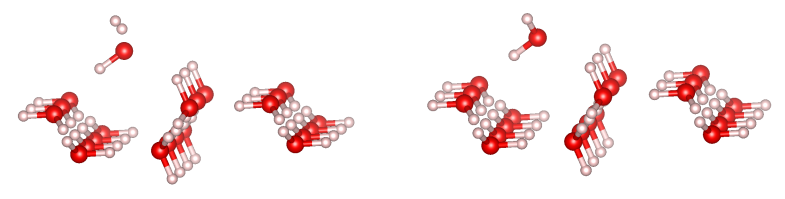

Fig. 4. Structures of the transition state (left) and final product of the $\mathrm{OH}$ first process for $\mathrm{H}_{2}+\mathrm{OH}_{\mathrm{ads}} \rightarrow \mathrm{H}_{2} \mathrm{O}_{\text {ads }}+\mathrm{H}$ on top of solid water ice.

The structure of the transition state is that represented on Fig. 4-left. The H-H distance of $\sim 0.82 \AA$ is larger than in the gas phase $(0.74 \AA)$. At the same time, the $\mathrm{OH}$ distance between the hydroxyl oxygen in $\mathrm{OH}$ and the nearest hydrogen of $\mathrm{H}_{2}$ is $\sim 1.33 \AA$. It is more than the equilibrium distance in $\mathrm{H}_{2} \mathrm{O}(1.00 \AA)$ but indicates the driving force towards the formation of the water molecule added to the subjacent ice represented on Fig. 4-right.

The critical parameters of the reaction mechanism $\Delta H^{\#}$ and $\Delta H$ are given in the last two columns of Table 3. The energetics of the reaction shows that, when $\mathrm{OH}$ is already adsorbed, the process is exothermic by approximately $-16 \mathrm{kcal} \mathrm{mol}^{-1}$ and presents a significant activation barrier of $\sim 4 \mathrm{kcal} \mathrm{mol}^{-1}$, this requiring a tunnel effect to reach the final products. Comparing these numbers with the preceding ones of the different clusters models leads to the conclusion that, in that case of $\mathrm{OH}$ adsorbed model, neither the activation barrier nor the exothermicity of the formation of $\mathrm{H}_{2} \mathrm{O}$ are significantly modified by the presence of the ice.

\section{Adding $\mathrm{OH}$ in the $\mathrm{H}_{2}$ adsorbed model}

In cold environments where icy grains are immersed in abundant molecular hydrogen, attack of the $\mathrm{OH}$ radical on $\mathrm{H}_{2}$ ads adsorbed has also been considered. The same set of calculations as above has been carried out on the small clusters, the ice bilayers and the solid ice $n\left(\mathrm{H}_{2} \mathrm{O}\right)=1,2,3,24,48$ and $\infty$ (Solid).

How the transition state energy $\Delta H^{\#}$ is sensitive to the molecule first adsorbed is shown in the first two lines of Table 4 where the results of the two mechanisms are reported for comparison.

The striking result of the $\mathrm{H}_{2}$ adsorbed model is that the process is really sensitive to the addition of $\mathrm{H}_{2} \mathrm{O}$ molecules and that there is no activation barrier as soon as $\mathrm{H}_{2} \mathrm{O}$ plays the role of support in the reaction. We find a hidden barrier whose depth below the reference energy increases with the number of supporting molecules to a limit of $\sim 10 \mathrm{kcal} \mathrm{mol}^{-1}$ for the solid model. This
Table 4. Transition state energies ${ }^{(*)} \Delta H^{\#}\left(\mathrm{kcal} \mathrm{mol}^{-1}\right)$ with respect to the energies of the reactants $(\mathrm{OH})_{\text {ads }},\left(\mathrm{H}_{2}\right)_{\text {ads }}$, and $\left(\mathrm{OH}+\mathrm{H}_{2}\right)_{\text {ads }}$ as a function of the number $(n)$ of $\mathrm{H}_{2} \mathrm{O}$ in the supporting cluster.

\begin{tabular}{lcccccc}
\hline \hline Cluster $(n)$ & 1 & 2 & 3 & $24(1-\mathrm{L})$. & $48(2-\mathrm{L})$. & $\infty$ \\
\hline $\mathrm{ER}(\mathrm{OH})$ & 4.7 & 4.6 & 4.5 & 4.1 & 3.5 & 4.1 \\
$\mathrm{ER}\left(\mathrm{H}_{2}\right)$ & -0.4 & -4.6 & -5.3 & -7.0 & -7.4 & -10.3 \\
$\mathrm{LH}\left(\mathrm{OH}+\mathrm{H}_{2}\right)$ & 5.4 & 5.0 & 4.9 & 4.7 & 3.9 & 5.1 \\
\hline
\end{tabular}

Notes. ${ }^{(*)}$ MPWB1K/aug-cc-pVTZ except for the 2-layers case where the basis set is $6-311++G(3 d f, 2 p)$.

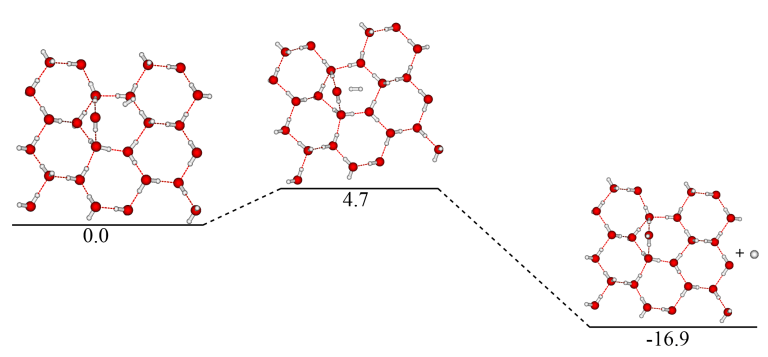

Fig. 5. Reaction profile for $\mathrm{H}_{2 \mathrm{ads}}\left({ }^{1} \Sigma^{+}\right)+\mathrm{OH}_{\mathrm{ads}}\left({ }^{2} \Pi\right) \rightarrow \mathrm{H}_{2} \mathrm{O}_{\mathrm{ads}}\left({ }^{1} \mathrm{~A}_{1}\right)+$ $\mathrm{H}\left({ }^{2} \mathrm{~S}\right)$ at the MPWB1K/aug-cc-pVTZ level in presence of a 1-layer of $\mathrm{H}_{2} \mathrm{O}$. Relative energies are given in $\mathrm{kcal} \mathrm{mol}^{-1}$.

means that no barrier should oppose to the formation of $\mathrm{H}_{2} \mathrm{O}$ in that case.

The reaction of $\mathrm{OH}$ with an $\mathrm{H}_{2}$ adsorbed on the ice surface is usually not considered because $\mathrm{E}\left(\mathrm{H}_{2}\right)_{\text {ads }}$ is supposedly too small for $\mathrm{H}_{2}$ to play a significant role. However, when looking carefully at this mechanism (see Table 4) it is obvious that this process can be efficient and is intrinsically different from that in which $\mathrm{OH}$, adsorbed on the surface is the target of the $\mathrm{H}_{2}$ attack. In short, the reaction is exothermic by approximately $-29 \mathrm{kcal} \mathrm{mol}^{-1}$ and there is no transition state on the way to the $\mathrm{H}_{2} \mathrm{O}$ formation.

\section{Reaction between $\mathrm{OH}$ and $\mathrm{H}_{2}$ both adsorbed on the ice}

The situation of multi adsorptions is largely plausible in the ISM context. Due to the one order of magnitude difference between $\mathrm{E}\left(\mathrm{OH}_{\mathrm{ads}}\right)$ and $\mathrm{E}\left(\mathrm{H}_{2}\right)_{\text {ads }}$ it is reasonable to expect the starting point of this mechanism to be lower than the one with only $\mathrm{OH}_{\mathrm{ads}}$ on the ice.

Simultaneous optimization of both $\mathrm{OH}$ and $\mathrm{H}_{2}$ on the supporting ice models, using the same protocol as before, leads to the new values of the transition states energies reported in line 3 of the updated Table 4 . The increase in the $\Delta H^{\#}$ values is systematic and illustrates the weak extra stabilization of the $\mathrm{LH}\left(\mathrm{OH}+\mathrm{H}_{2}\right)_{\text {ads }}$ starting complex. This difference would have been close to $\mathrm{E}\left(\mathrm{H}_{2}\right)_{\text {ads }}$, if there were no structural relaxation of the system. The energy profile is shown in Fig. 5.

\section{Summary and final remarks}

The aim of this theoretical work was to shed some light, at the molecular scale, on the feasibility of the

$\mathrm{H}_{2}+\mathrm{OH} \rightarrow \mathrm{H}_{2} \mathrm{O}+\mathrm{H}$

reaction proposed by Tielens \& Hagen (1982) as one of the mechanisms at the origin of the formation of interstellar ices. In view of the resilience of the ice confronted to the radiation 
field we wondered about the influence of the water support on its own reconstruction as well as the influence of the environmental conditions. Indeed this reaction is particularly important in regions of high density where the temperature is low $(10-30 \mathrm{~K})$ so that the sticking of $\mathrm{H}_{2}$ on the surfaces of grains becomes critical to astrochemical modeling (Wakelam et al. 2016; Ruaud et al. 2016).

To address the question of the ice reconstruction we used a multistep approach seeking continuity between water aggregates and solid state as the number $n$ of $\mathrm{H}_{2} \mathrm{O}$ molecules in the reaction support grows to infinity. It is well-known indeed that a single computational methodology cannot accurately describe the same chemical process at different molecular scales. Small clusters can be treated by the most sophisticated post HartreeFock methods but they can only provide accurate results for case studies that cannot describe a real ice support. For more realistic systems of larger dimensions we turned to DFT known to deliver reliable results for such systems. Our last step of calculations considers the solid state situation taking a surface of infinite dimension to close the question of the surface extension.

Considering the binding energies in Table 2 we may expect three different mechanisms at the molecular level, $\mathrm{ER}(\mathrm{OH})$ and $\mathrm{ER}\left(\mathrm{H}_{2}\right)$ of Eley-Rideal type and $\mathrm{LH}\left(\mathrm{OH}+\mathrm{H}_{2}\right)$ of LangmuirHinschelwood type.

The last situation is essentially adapted to large clusters and solid state, then a plausible alternative in the ISM context. Due to the one order of magnitude difference between $\mathrm{E}\left(\mathrm{OH}_{\mathrm{ads}}\right)$ and $\mathrm{E}\left(\mathrm{H}_{2}\right)_{\text {ads }}$ we find the starting point of the LH mechanism to be lower than the one with only $\mathrm{OH}_{\mathrm{ads}}$ on the ice, the consequence of which is an increase in the barrier opposing the reconstruction of the ice. The energy profiles of the three mechanisms are presented together with the Langmuir-Hinshelwood mechanism in which both $\mathrm{OH}$ and $\mathrm{H}_{2}$ are adsorbed (Fig. 6) taking the energy of the non interacting system $\left[\mathrm{OH}, \mathrm{H}_{2},\left(\mathrm{H}_{2} \mathrm{O}\right)_{n}\right]$ as arbitrary reference.

The remarkable result is that, whatever the theoretical model, the activation barrier is still present on the energy profile for the reaction

$\mathrm{H}_{2}+\mathrm{OH}_{\mathrm{ads}} \rightarrow \mathrm{H}_{2} \mathrm{O}_{\mathrm{ads}}+\mathrm{H}$

of $\mathrm{H}_{2}$ on an adsorbed $\mathrm{OH}$ with a value of $\sim 4 \mathrm{kcal} \mathrm{mol}^{-1}$. The presence of a water solid support seems to have no influence at all on the process at the molecular level. The result seems consistent with both the early work by Baulch et al. (1984) and the recent study of Meisner et al. (2017) who also argued that only $\mathrm{OH}$ adsorption should be considered in view of its large value. Nevertheless, in opposition to the common paradigm, and knowing the high density of $\mathrm{H}_{2}$ in many regions of the ISM, we considered that the coverage of the ice by $\mathrm{H}_{2}$ is also a reasonable assumption taking account the relative abundances of these two species $\left(\mathrm{H}_{2} / \mathrm{OH}\right.$ certainly $\left.\gg 10\right)$. We found a completely different energetic profile of the reaction

$\mathrm{OH}+\mathrm{H}_{2}$ ads $\rightarrow \mathrm{H}_{2} \mathrm{O}_{\text {ads }}+\mathrm{H}$

when $\mathrm{OH}$ reacts with an $\mathrm{H}_{2}$ adsorbed on the ice. Namely, that there is no activation energy to prevent the pursuit of the $\mathrm{H}_{2} \mathrm{O}$ synthesis. It must be stressed that this result is valid whatever the size of the ice support, cluster of limited size or solid surface of infinite dimensions which should be equally correct for both porous or crystalline ices. The solid state approach, designed to bypass the possible artifacts (if any) coming from the use of models limited in size, provides a robust confirmation of no barrier at all opposing the reaction on the surface.

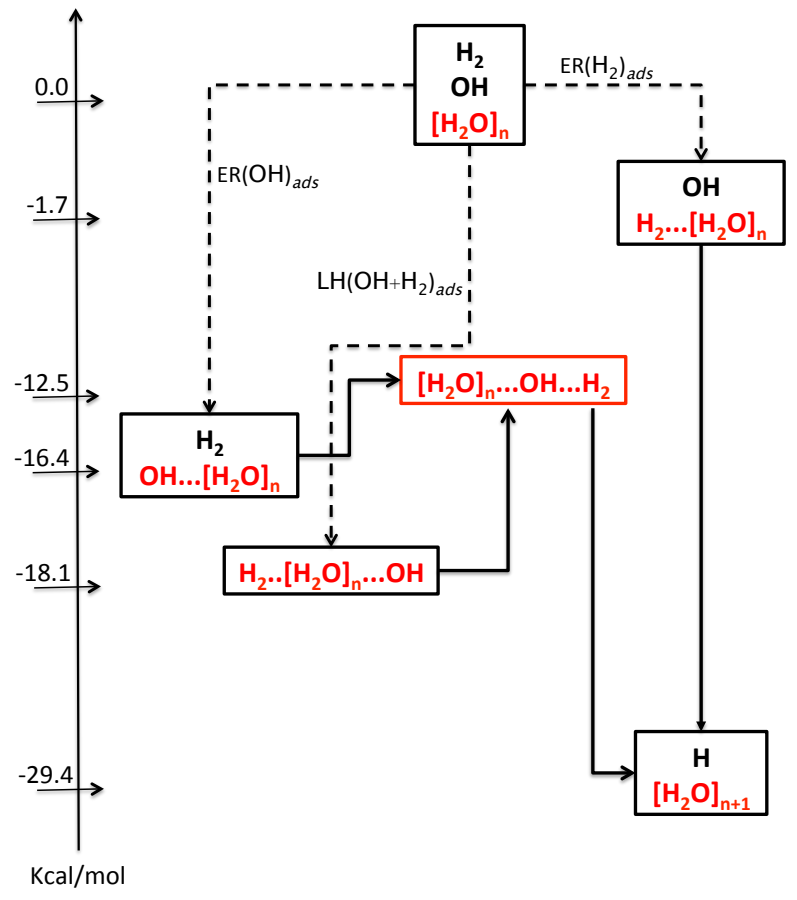

Fig. 6. Schematic profiles of the three mechanisms $\operatorname{ER}(\mathrm{OH})_{\mathrm{ads}}$, $\mathrm{ER}\left(\mathrm{H}_{2}\right)_{\text {ads }}$ and $\mathrm{LH}\left(\mathrm{OH}+\mathrm{H}_{2}\right)_{\text {ads }}$ leading to reconstruction of the cristalline ice. Dotted lines follow the adsorption path prior to the reaction. The solid species are in red characters within boxes. Energies are given with respect to the non interacting system.

In short, when $\mathrm{H}_{2}$ reacts with $\mathrm{OH}$ adsorbed on the ice, $\Delta H^{\#}$ is in the range of $4.1 \pm 0.6 \mathrm{kcal} \mathrm{mol}^{-1}$ whatever the ice support, small cluster or solid water ice. It is totally different when it is $\mathrm{OH}$ that reacts with $\mathrm{H}_{2}$ adsorbed on the ice. The formation of $\mathrm{H}_{2} \mathrm{O}$ becomes a no-barrier process as soon as $\mathrm{H}_{2} \mathrm{O}$ is implied.

The only problem with the " $\mathrm{H}_{2}$ as a target" hypothesis is that of the temperature of the grain ice mantles. For very low temperature, $\sim 10 \mathrm{~K}$, several layers of $\mathrm{H}_{2}$ are deposited on the ice surface. The deepest one is attached to the ice by an energy between $\sim 440 \mathrm{~K}$ (value estimated by Cuppen \& Herbst (2007) from a number of experiments) and our theoretical value of $\sim 850 \mathrm{~K}$ (this work). The upper layers are pure Van der Waals stacking of hydrogen on top of hydrogen, with a binding energy in the order of $23 \mathrm{~K}$ according to Cuppen \& Herbst (2007).

The ratio of $\sim 2$ between the estimated binding energies of $\mathrm{H}_{2}$ on the experimental ice and the value obtained in the solid state calculation may have several origins. One bias may come from the large distribution of energies found in TPD measurements (Dulieu et al. 2005; Hornekr et al. 2005), possibly influenced by the weak adsorption energy of $\mathrm{H}_{2}$ onto an important number of $\mathrm{H}_{2}$ layers piled on top of each other compared to a single layer of $\mathrm{H}_{2}$ in contact with the ice mantle. Another bias may be due to the calculation model itself in which we consider an isolated $\mathrm{H}_{2}$ on top of a crystalline ice. But, as shown by the values listed in Table 4, none of these eventual drawbacks could modify the conclusion that reaction of $\mathrm{OH}$ on an adsorbed $\mathrm{H}_{2}$ is a no barrier process.

\section{Conclusion}

The present theoretical study of the reaction initially proposed by Tielens \& Hagen more than $30 \mathrm{yr}$ ago for the formation of the ice

$\mathrm{H}_{2}+\mathrm{OH} \rightarrow \mathrm{H}_{2} \mathrm{O}+\mathrm{H}$ 
shows that this process is efficient once a first layer of ice is present to assist in the growth of the ice mantle. Another important aspect is that this mechanism should be equally efficient in the reconstruction of the ice layers following the damages created by irradiations. This either in the Eley-Rideal process in which only $\mathrm{OH}$ is adsorbed or in the composite Langmuir-Hinschelwood process in which $\mathrm{OH}$ and $\mathrm{H}_{2}$ are both attached to the ice before reaction. In this context, there is an activation barrier opposing the formation of $\mathrm{H}_{2} \mathrm{O}$ that requires tunneling to proceed, the barrier being higher when the $\mathrm{OH}$ and $\mathrm{H}_{2}$ reagents are adsorbed. In a low temperature environment $(\sim 10-30 \mathrm{~K})$ the $\mathrm{OH}+\mathrm{H}_{2 \text { ads }}$ should give $\mathrm{H}_{2} \mathrm{O}$ directly i.e. without activation barrier whereas the conventional $\mathrm{H}_{2}+\mathrm{OH}$ tunneling mechanisms should prevail above $\sim 50 \mathrm{~K}$. For intermediate situations these three mechanisms should cooperate in the reconstruction of the ice on the interstellar grains.

In the end, the type of catalytic reactions shown here on the example of the formation/reconstruction of the ice is probably more general. These results should be a motivation to carry out theoretical as well as experimental in-depth studies of bimolecular reactions at the surface of interstellar solids. This especially when the two reagents implied have adsorption energies whose difference is in the range of an order of magnitude or more.

Acknowledgements. This work was supported by CNRS national program PCM (Physics and Chemistry of the Interstellar Medium) and COST Action CM 805, (The Chemical Cosmos). P.R. acknowledges the funding support from the Spanish Ministerio de Economia Industria y Competitividad (Grant AYA201787515-P) and the Junta de Castilla y León (Spain; Grant No. VA010G18).

\section{References}

Amiaud, L., Fillion, J. H., Dulieu, F., Momeni, A., \& Lemaire, J. L. 2015, PCCP 17, 30148

Bartlett, R. J., \& Shavitt, I. 1977, Chem. Phys. Lett., 50, 190

Baulch, D. L., Cox, R. A., Hampson, R. F., et al. 1984, J. Phys. Chem. Ref. Data, 13,1259

Becke, A. D. 1993, J. Chem. Phys., 98, 1372

Bernal, J. D., \& Fowler, R. H. 1933, J. Chem. Phys., 1, 515

Bertin, M., Doronin, M., Fillion, J. H., et al. 2017, A\&A, 598, A18

Boogert, A. C. A., Pontoppidan, K. M., Knez, C., et al. 2008, ApJ, 678, 985

Buch, V., Bauerecker, S., Devlin, J. P., Buck, U., \& Kazimirski, J. K. 2004, Int. Rev. Phys. Chem., 23, 375

Burke, D. J., \& Brown, W. 2010, J. Phys. Chem. Chem.Phys., 12, 5947

Bussolin, G., Casassa, S., Pisani, C., \& Ugliengo, P. 1998, J. Chem. Phys., 108, 9516

Calatayud, M., Courmier, D., \& Minot, C. 2003, Chem. Phys. Lett., 369, 287

Casassa, S., \& Pisani, C. 2002, J. Chem. Phys., 116, 9864

Casassa, S., Calatayud, M., Doll, K., Minot, C., \& Pisani, C. 2005, Chem. Phys. Lett., 409, 110

Chen, J., Xu, X., Xu, X., \& Zhang, D. H. 2013, J. Chem. Phys., 138, 154301

Cuppen, H. M., \& Herbst, E. 2007, ApJ, 668, 294

Cuppen, H. M., Ioppolo, S., Romanzin, C., \& Linnartz, H. 2010, Phys. Chem. Chem. Phys., 12, 12077

Dartois, E., Cox, P., Roelfsema, P. R., et al. 1998, A\&A, 338, L21

Doronin, M., Bertin, M., Michaut, X., Philippe, P., \& Fillion, J.-H. 2015, J. Chem. Phys., 143, 084706

Dulieu, F., Amiaud, L., Baouche, S., et al. 2005, Chem. Phys. Lett., 404, 187

Eddington, A. S. 1926, The Observatory, 49, 304

Frisch, M., Trucks, G., Schlegel, H., et al., 2013 Gaussian 09, Revision D.01 (Gaussian, Inc., Wallingford CT)

Gibb, E., Whittet, D. B., Boogert, A. A., \& Tielens, A. G. G. M. 2004, Ap\&SS, 151,35

Govers, T. R., Mattera, L., \& Scoles, G. 1980, J. Chem. Phys., 72, 5446

Grimme, S., Antony, J., Ehrlich, S., \& Krieg, H. 2010, J. Chem. Phys., 132, 154104

Hasegawa, T. I., \& Herbst, E. 1993, MNRAS, 263, 589.

d'Hendecourt, L., Allamandola, L. J., \& Greenberg, J. M. 1985, A\&A, 152, 130

Hirsch, T. K., \& Ojamae, L. 2004, J. Phys. Chem. B, 108, 15856

Hornekr, L., Baurichter, A., Petrunin, V. V., et al. 2005, J. Chem. Phys., 122, 124701

Ioppolo, S., Cuppen, H. M., Romanzin, C., van Dishoeck, E. F., \& Linnartz, H. 2010, Phys. Chem. Chem. Phys., 12, 12065
Kresse, G., \& Hafner, J. 1993, Phys. Rev. B, 48, 13115

Kresse, G., \& Hafner, J. 1994, Phys. Rev. B, 49, 14251

Kuo, J. L., \& Singer, S. J. 2003, Phys. Rev. E, 67, 016114

Lamberts, T., \& Kästner, J. 2017, ApJ, 846, 43

Lattelais, M., Bertin, M., Mokrane, H., et al. 2011, A\&A, 532, A12

Lattelais, M., Pauzat, F., Ellinger, Y., \& Ceccarelli, C. 2015, A\&A, 572, A62

Lynch, B. J., Fast, P. L., Harris, M., \& Truhlar, D. G. 2000, J. Phys. Chem. A, 104,4811

Meisner, J., Lamberts, T., \& Kästner, J. 2017, Earth Space Sci., 1, 399

Merrill, K. M., Russell, R. W., \& Soifer, B. T. 1976, Icarus, 207, 763

Mokrane, H., Chaabouni, H., Accolla, M., et al. 2009, ApJ, 705, L195

Mousis, O., Ronnet, T., Brugger, B., et al. 2016, ApJ, 823, L41

Oba, Y., Watanabe, N., Hama, T., et al. 2012, ApJ, 749, 67

Oberg, K. I., Boogert, A. C. A., Pontoppidan, K. M., et al. 2011, ApJ, 740, 109

Pan, D., Liu, L. M., Tribello, G. A., et al. 2010, J. Phys. Condens. Matter, 22, 074209

Pauzat, F., Lattelais, M., Ellinger, Y., \& Minot, C. 2011, MNRAS, 412, 2729

Perets, H. B., Biham, O., Manico, G., et al. 2005, ApJ, 627, 850

Raghachavari, K., Trucks, G. W., Pople, J. A., \& Head-Gordon, M. 1989, Chem. Phys. Lett., 157, 479

Redondo, P., Pauzat, F., Ellinger, Y., \& Markovits, A. 2020, A\&A, 638, A125

Ruaud, M., Wakelam, V., \& Hersant, F. 2016, MNRAS, 459, 3756

Tielens, A. G. G. M., \& Hagen, W. 1982, A\&A, 114, 245

Vidali, G., Ihm, G., Kim, H. Y., \& Cole, M. 1991, Surf. Sci. Rep., 12, 133

Wakelam, V., Ruaud, M., Hersant, F., et al. 2016, A\&A, 594, A35

Whittet, D. C. B., Bode, M. F., Longmore, A. J., et al. 1988, MNRAS, 233, 321

Zao, Y., \& Truhlar, D. G. 2004, J. Phys. Chem. A, 108, 6908

\section{Appendix A: Solid state modeling}

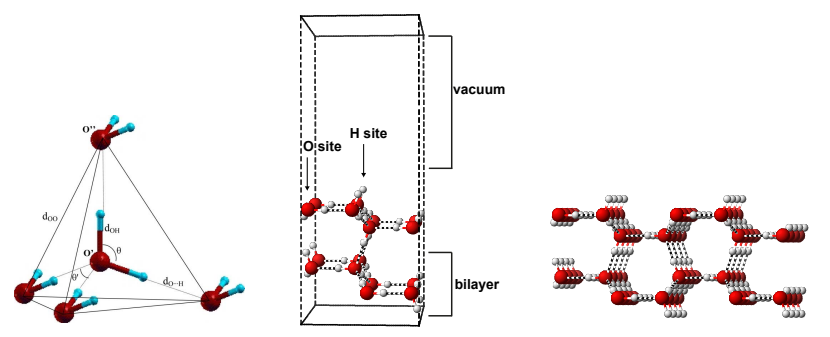

Fig. A.1. Bernal-Fowler arrangement (left) and Side views of the boat structure of hexagonal apolar ice unit cell (center) and (right) with two strata. It should be noted that one stratum of ice is composed of one crystallographic bi-layer of $\mathrm{H}_{2} \mathrm{O}$ molecules.

Modeling solid water has attracted increasing attention in the past fifteen years (Casassa \& Pisani 2002; Calatayud et al. 2003; Kuo \& Singer 2003; Hirsch \& Ojamae 2004; Casassa et al. 2005). Levering on the conclusions of these works and our own experience (Lattelais et al. 2011, 2015; Mousis et al. 2016; Bertin et al. 2017), we took a periodic representation of solid water in the form of hexagonal ice $I_{\mathrm{h}}$ composed of bi-layers of water molecules. To be consistent with the above study, the ice surface was described by the (0001) Miller indices. This choice of an apolar ice satisfying the Bernal \& Fowler (1933) arrangement is computationally justified because only apolar structures can generate slabs that are stable (Bussolin et al. 1998), reproduce the bulk properties, and have a balanced distribution of alternate hydrogen and oxygen sites at their surfaces (for more details, see Casassa et al. 2005).

Here, the basal plane dimensions (corresponding to the surface interacting with the adsorbate) are $a=7.145 \AA$ and $b=8.73 \AA$. The third dimension of the cell $c$ was determined so that there is no interaction between molecules generated by a translation perpendicular to the ice surface.

More insight in the Vienna $\mathrm{Ab}$ initio Simulation Package online ${ }^{3}$.

3 https://cms.mpi.univie.ac.at/vasp/vasp/Hartree_Fock_ HF_type_hybrid_functional_calculations.html 